DOI: 10.17707/AgricultForest.64.1.17

\begin{abstract}
Mehdi ABDOLAHI, Farid SHEKARI*, Jalal SABA and Esmaeil ZANGANI ${ }^{1}$
\end{abstract}

\title{
SEED PRIMING WITH SALICYLIC ACID ENHANCED GAS EXCHANGES PARAMETERS AND BIOLOGICAL YIELD OF WHEAT UNDER LATE SOWING DATE
}

\begin{abstract}
SUMMARY
This experiment was done to evaluate the effect of two planting dates and salicylic acid (SA) on wheat photosynthesis. Wheat seeds, cv. Alvand, primed with SA $(0,400,800,1200,1600,2000$ and $2400 \mu \mathrm{M})$ at two planting dates (recommended planting date, 23 October, and late planting date, 22 November). Gas exchange parameters were measured in three growth stages (tillering, heading and grain filling). The highest and lowest rate of photosynthesis $\left(P_{\mathrm{N}}\right)$, stomatal conductance $\left(g_{\mathrm{s}}\right)$ and transpiration rate $(E)$ of plants were observed in heading and grain filling stages, respectively. Seed pretreatment with SA enhanced photosynthetic parameters and carboxylation efficiency (CE), but, intercellular $\mathrm{CO}_{2}$ concentration and water use efficiency (WUE) reduced by application of SA. It seems that application of SA had more effects on $g_{s}$ and $E$ than $P_{\mathrm{N}}$. Among growth stages, the highest value of WUE was found in tillering and lowest in heading stage. Priming with SA compensated late sowing effects on plants $P_{N}$. Chlorophyll content, chlorophyll a/b ratio and CCI values significantly increased in SA treated plants. Results show that priming with SA may reduce ameliorative effects of late sowing on wheat plant biomass production. Among SA concentrations, $1200 \mu \mathrm{M}$ had highest value in both planting dates.
\end{abstract}

Keywords: Carboxylation efficiency, Chlorophyll content, Photosynthesis rate, Water use efficiency.

\section{INTRODUCTION}

Photosynthesis and related gas exchange parameters influenced by many internal and external factors. For example, it is reported leaf ontogeny, heterophylly and position (Hejnak et al., 2014), age (Wang et al., 2014), seasonal changes and conditions (Ribeiro et al., 2009), sink effect (Nebauer et al., 2011) have considerable effects on photosynthesis rate and its regulation. Level of leaf development and/or morphological and anatomical stage of plant may influence photosynthesis rate (Hejnak et al., 2014). Also, environmental history of leaves affect their photosynthetic development (Fitter and Hey, 2012).

\footnotetext{
${ }^{1}$ Mehdi Abdolahi, Farid Shekari*(corresponding author: shekari@znu.ac.ir), Jalal Saba and Esmaeil Zangani, Department of Agronomy, Faculty of Agriculture, University of Zanjan, IRAN Notes: The authors declare that they have no conflicts of interest. Authorship Form signed online.
} 
Sowing date is a key factor on plants productivity potential and has deep effect on crop yield. It can by influence on plant tissues age change photosynthetic capacity of plants.

Salicylic acid (SA) is an endogenous growth regulator with phenolic structure, which participates in the regulation of different physiological and biochemical processes in plants (Raskin, 1992) and acts as an important signaling molecule (Nazar et al. 2011). SA, might play a role in $g_{s}$ (Janda et al., 2014), photosynthesis (Fariduddin et al. 2003), and stomatal closure (Poor and Tari, 2012). Nazar et al. (2011) reported in mungbean SA increased photosynthesis under normal condition and alleviated salt effects on photosynthesis. These protective effects may be related with nitrogen and sulfur assimilation metabolism. Spraying of maize plants with SA led to increase photosynthesis, pigment content and growth rate (Khodary, 2004).

At many parts of Iran, because of unfavorable weather condition, rotation of wheat after late potato or maize cultivars and/or large planting areas, planting date may delay until late of autumn and these situations leads to weakly growth of seedlings or fail in establishment by cold stress damage. On the other hand, it is possible later growth and photosynthesis of plants after winter affected by pervious growth stages and growth history of plants. The aim of our experiment was to study the effects of two different sowing dates on gas exchange parameters and biological yield of wheat and possibility of ameliorative effects of seed priming by SA on these parameters.

\section{MATERIAL AND METHODS}

Seeds of wheat (Triticum aestivum L., cv Alvand) obtained from seed bank of faculty of Agriculture, University of Zanjan. Seed moisture content was 8.54\% (based on dry weight). For all treatments, selected healthy seeds were used in same numbers.

\section{Salicylic acid seed treatments}

For seed priming, SA solutions prepared in six levels, including 400, 800, 1200, 1600, 2000 and $2400 \mu \mathrm{M}$. For each treatment SA powder (Merck, Darmstadt. Germany) weighted separately and solved in 5 cc ethanol and shaken well. Then solution added to 3 litter distilled water. The ratio of seed to solute was 1:5 (based on weight). Seeds submerged for 12 hours at $4{ }^{\circ} \mathrm{C}$. Then, seeds exposed to airflow and air-dried. Non primed seeds, were used as control treatment.

\section{Planting and cultivation:}

Seeds planted in two planting dates: 23 October (as conventional planting date in Zanjan province) and 22 November (as late planting date) of 2010 in research station of University of Zanjan $\left(36^{\circ} 40^{\prime} \mathrm{N} ; 48^{\circ} 24 \mathrm{E}\right.$ and $1610 \mathrm{~m}$ from sea level). In general, Iran has arid and semi-arid climate and the major precipitation occur from October to June (Table 1). The coldest month mainly occurs in January and cold weather and frost happen mid to end of November to March (Alijani, 2006). The day after planting plots irrigated and irrigation continued 
until frizzing temperature was appeared. In spring from $4^{\text {th }}$ of May irrigation again started normally each week. According to soil analysis $40 \mathrm{~kg} / \mathrm{ha}$ phosphate in form of phosphate ammonia before planting and $80 \mathrm{~kg} /$ ha nitrogen in form of urea in two times (after seedlings emergence and before stem elongation) were added to field.

Table 1. Mean temperature of 10 years (from 1999-2009) and the experiment year (2009-10) of Zanjan region.

\begin{tabular}{|c|c|c|c|c|}
\hline & \multicolumn{2}{|c|}{$2009-2010$} & \multicolumn{2}{c|}{$1999-2009$} \\
\hline Months & Max & Min & Max & Min \\
\hline October & 27.2 & -1.6 & 26.8 & 0.3 \\
\hline November & 18.8 & -7 & 18.4 & -8 \\
\hline December & 11.8 & -8.4 & 12.4 & -13.9 \\
\hline January & 14.4 & -15.4 & 10.5 & -17.3 \\
\hline February & 17.4 & -12 & 12.8 & -11.8 \\
\hline March & 25.8 & -4.2 & 20.3 & -7.6 \\
\hline April & 23 & 0.2 & 25 & -2.6 \\
\hline May & 29 & 1.2 & 29 & 2.1 \\
\hline June & 35.6 & 9 & 34.4 & 5.9 \\
\hline July & 40 & 10 & 36.1 & 10.1 \\
\hline
\end{tabular}

\section{Gas exchange}

Gas exchange parameters in three growth stages (tillering, heading and grain filling) were recorded. Photosynthesis rate $\left(P_{N}\right)$, stomatal conductance $\left(\mathrm{g}_{\mathrm{s}}\right)$, transpiration rate $(E)$ and intercellular $\mathrm{CO}_{2}$ concentration $\left(\mathrm{C}_{\mathrm{i}}\right)$ were measured using a portable open-system infrared gas analyzer ( $L C i$, ADC Bioscientific Ltd., Hoddesdon, UK).

All measurements were done in 10-12 a.m. and light intensity equivalent to $1200-1800 \mu \mathrm{mol}$ photons. ${ }^{-2}$. It is reported that $\mathrm{g}_{\mathrm{s}}$ during $10 \mathrm{am}$ to $1 \mathrm{pm}$ no significant changes (Clark and Mc ciag, 1982) and also at this light intensity $g_{s}$ reaches to a maximum. Before measuring, apparatus started for 10 minutes. For measuring gas exchange parameters, same leaves of plants in each treatment placed in chamber glass clamp of apparatus. Data recorded after 45 seconds as the chamber conditions receive as stable state.

Photosynthetic water use efficiency (WUE) and Carboxylation efficiency (CE) also was calculated based on the following formula (Ashraf et al; 2002):

Photosynthetic water use efficiency $\left(\mathrm{WUE}_{\mathrm{b}}\right)=P_{N} / E$

Carboxylation efficiency (CE) $=P_{N} / C_{i}$

\section{Chlorophyll content index (CCI)}

Chlorophyll content index was measured by a chlorophyll meter handheld device (CCM-200 ADC, UK) in all three stages from 10 randomly selected plants'. Middle part of same leaves was used for this reason. In tillering fourth or fifth leaf was measured and in two later growths stages the flag leaves were measured. 


\section{Chlorophyll a, b and total:}

Chlorophyll content of flag leaf in anthesis stage determined by method which described by Meidner (1984). The statistical analysis was done by using software MSTATC and SPSS. Means comparison was done by Duncan multiple test.

\section{Photosynthesis rate $\left(P_{\mathrm{N}}\right)$}

\section{RESULTS AND DISCUSSION}

In both planting date and in all three growth stages priming significantly increased $P_{\mathrm{N}}$ rate. Except to grain filling stage, in tillering and heading stages early planted wheat had higher $P_{\mathrm{N}}$ compared to late plated wheat (Table 2, 3 and 4). The highest $P_{N}$ in both planting date was observed in heading stage, then in tillering stage. In both planting dates, a decline was found in $P_{\mathrm{N}}$ in grain filling stage compared to heading stage (Table 3 and 4). In addition, at grain filling stage, all prim treatments in late planting had higher $P_{\mathrm{N}}$ compared to conventional planting date. It seems that, plants in the late planting treatment had younger tissues than conventional planting date. Also, it seems that, in grain filling compared to heading and tillering stages leaves was matured and more aged and therefore, had lower capacity in photosynthesis. In addition, it is possible in heading and pollination stage there was a higher demand for photoassimilates and may be it is a reason for the highest $P_{\mathrm{N}}$ in this stage. As mentioned above, priming with SA leads to increasing $P_{\mathrm{N}}$. Among priming treatments, priming with $1200 \mu \mathrm{M}$ concentration, in both planting date and in all three growth stages had the highest rate of photosynthesis (Table 2, 3 and 4).

Enhancement activity of carbonic anhydrase in leaves of mustard (Fariduddin et al. 2003) and Rubisco in maize (Khodary, 2004) by application of SA was reported. Also, protection of the photosynthetic apparatus has also been reported in SA treated tomato plants (Poor et al., 2011). On the other hand, higher concentrations of SA may have prevention effects on photosynthesis (Janda et al., 2014). Our results show that in concentrations over $1200 \mu \mathrm{M}$ a decline observed in $P_{\mathrm{N}}$.

\section{Stomatal conductivity $\left(\boldsymbol{g}_{s}\right)$}

Seed priming increased $g_{\mathrm{s}}$ in three stages and in both planting dates. Also, it compensated the reduction in $g_{\mathrm{s}}$ in late planting in all growth stages. $g_{\mathrm{s}}$ in both planting and in the heading stage reached highest value in $1200 \mu \mathrm{M}$ SA compared to other SA treatments (Table 2, 3 and 4). In comparison of two planting dates, $g_{\mathrm{s}}$ in late planting had higher value than conventional planting date in grain filling stage. Among growth stages the lowest $g_{\mathrm{s}}$ were observed in grain filling and highest in heading stage. The high ratio of $g_{\mathrm{s}}$ in heading compared to grain filling and tillering may be due to young age of leaves and demand of atmosphere for transpiration, respectively.

In contrast, in tillering stage as environment was cool than two other stages the $g_{\mathrm{s}}$ showed lower amounts. It is reported exogenous application of SA in wheat promoted growth and yield which associated with increased 
photosynthesis capacity and $g_{\text {s. }}$ (Arfan et al. 2007).

\section{Transpiration rate $(E)$}

The highest $E$ was observed in the heading and the lowest in tillering and grain filling stages. Priming increased $E$ in all different growth stages. Among priming treatments, $1200 \mu \mathrm{M}$ had the most effects on $E$ in both planting dates and all growth stages. In general, priming by $\mathrm{SA}$ improved the $E$ values and enhanced its values compared to control treatments (Table 2, 3 and 4). Higher rate of transpiration may be related to increasing root development or efficient uptake of water by increasing root length and density which reported by Sandoval-Yepiz (2004) and Abdolahi and Shekari (2013). The lower value of $E$ in tillering and grain filling stages may be due to cool temperature in early spring; and maturation of leaves, and therefore, reduction in capacity of leaves transpiration from aged plants, respectively.

\section{Intercellular $\mathrm{CO}_{2}$ concentration $\left(C_{i}\right)$}

$C_{i}$ was decreased by application of SA in three stages and two planting date and control treatments had higher $C i$ values. Among three growth stages the lowest $C_{i}$ was found in heading stage and two other stages had higher values than mentioned stage (Table 2, 3 and 4). In general, $1200 \mu \mathrm{M}$ of SA showed the lowest rate in all treatments expect tillering stage. In tillering stage 400 and 800 $\mu \mathrm{M}$ and in grain filling $400 \mu \mathrm{M}$ of SA had not significant differences with control treatments. In tillering stage 2000 and $1600 \mu \mathrm{M} \mathrm{SA}$ in conventional and late planting had lowest $C i$ respectively (Table 2, 3 and 4). Lower rate of $C_{i}$ in priming treatments than control treatments may due to higher $\mathrm{CE}$ or higher performance in assimilation of $\mathrm{CO}_{2}$ in photosynthesis process. It was reported foliar spraying of $0.5-2.5$ mmol. $\mathrm{L}^{-1}$ of SA on cucumber seedlings before the low temperature stress increased the leaf $P_{\mathrm{N}}, g_{\mathrm{s}}, E, \Phi$ PSII, Fv/Fm, while decreased the $\mathrm{Ci}$ (Liu et al., 2009).

\section{Carboxylation efficiency (CE)}

Priming significantly increased $\mathrm{CE}$ in all growth stages and two planting dates (Table 2, 3 and 4). The highest value of CE was observed in heading stage and conventional planting date and the lowest was observed in grain filling stage and conventional planting date. Since, the values of $P_{N}$ were higher and $C i$ was lower in this stage, it is reasonably highest amounts of CE found in this stage. In heading stage, priming in highest values improved CE to $42 \%$ and $26 \%$ on the first and second planting date compared to control treatments, respectively. At all phenological stages, $1200 \mu \mathrm{M}$ treatment, had the highest CE rate. High CE in priming treatments suggests more effective assimilation of carbon in these treatments relative to control treatments. Zhen et al. (2010) reported Chrysanthemum plants treatment with ASA increased CE, so caused reduction in $C_{i}$ under low temperature stress with lower light intensity. They suggested that more tolerance to cold stress correlated with higher values of CE, $g_{\mathrm{s}}$ and $P_{\mathrm{N}}$.

\section{Photosynthetic water use efficiency (WUE)}

The lowest rate of WUE was observed in heading stage and the highest was observed in tillering stage (table 2, 3 and 4). 
Table 2. Effect of priming by salicylic acid (SA) on, net photosynthetic rate (PN), stomatal conductance (gs), Transpiration Rate (E), intercellular $\mathrm{CO}_{2}$ concentration (Ci), Carboxylation efficiency (CE), and photosynthetic water use efficiency (WUE) in tillering stage in wheat plants.

\begin{tabular}{|c|c|c|c|c|c|c|c|}
\hline $\begin{array}{c}\text { Planting } \\
\text { date }\end{array}$ & $\begin{array}{c}\text { Priming } \\
{[\mu \mathrm{M}]}\end{array}$ & $\begin{array}{c}P_{N} \\
{\left[\mu \mathrm{mol}^{-2}\left(\mathrm{CO}_{2}\right)\right.} \\
\left.\mathrm{m}^{-2} \mathrm{~s}^{-1}\right] \\
\end{array}$ & $\begin{array}{c}g_{s} \\
{\left[\mathrm{mmol} \mathrm{m}_{2}^{-1} \mathrm{~m}^{-}\right.}\end{array}$ & \begin{tabular}{|c|}
$E$ \\
{$\left[\begin{array}{c}\mathrm{mmol}^{-}\left(\mathrm{H}_{2} \mathrm{O}\right) \\
\left.\mathrm{m}^{-2} \mathrm{~s}^{-1}\right]\end{array}\right.$} \\
\end{tabular} & $\begin{array}{c}\mathrm{Ci} \\
{\left[\mu \mathrm{mol}\left(\mathrm{CO}_{2}\right)\right.} \\
\left.\operatorname{mol}(\text { air })^{-1}\right]\end{array}$ & $\begin{array}{c}\mathrm{CE} \\
{\left[\mathrm{mmol}^{-2}\left(\mathrm{CO}_{2}\right)\right.} \\
\left.\mathrm{m}^{-2} \mathrm{~s}^{-1}\right] \\
\end{array}$ & $\begin{array}{c}\text { WUE } \\
{\left[\mu m o l\left(\mathrm{CO}_{2}\right)\right.} \\
\left.\mathrm{mmol}^{-1}\left(\mathrm{H}_{2} \mathrm{O}\right)\right]\end{array}$ \\
\hline $\begin{array}{l}\text { Stress } \\
\text { period }\end{array}$ & & & & & & & \\
\hline \multirow{7}{*}{$\begin{array}{c}\text { Recomm } \\
\text { ended } \\
\text { planting } \\
\text { date }\end{array}$} & Control & $13.472^{\mathrm{bc}}$ & $175^{d}$ & $3.572^{\mathrm{h}}$ & $233.5^{\mathrm{a}}$ & $57.82^{\text {efg }}$ & $3.77^{\mathrm{a}}$ \\
\hline & 400 & $15.403^{\mathrm{abc}}$ & $245^{c}$ & $4.375^{\text {cde }}$ & $233.5^{\mathrm{a}}$ & $65.98^{\text {bcd }}$ & $3.52^{\mathrm{abc}}$ \\
\hline & 800 & $16.712^{\mathrm{abc}}$ & $292.5^{b}$ & $5.463^{\mathrm{a}}$ & $232.5^{\mathrm{a}}$ & $83.04^{\mathrm{a}}$ & $3.06^{\mathrm{abc}}$ \\
\hline & 1200 & $18.155^{\mathrm{a}}$ & $325^{a}$ & $5.84^{\mathrm{a}}$ & $216.75^{\mathrm{b}}$ & $83.175^{a}$ & $3.396^{\mathrm{abc}}$ \\
\hline & 1600 & $16.862^{\mathrm{a}}$ & $250^{c}$ & $4.927^{\mathrm{b}}$ & $216.5^{b}$ & $69.183^{\mathrm{bc}}$ & $3.058^{\mathrm{bc}}$ \\
\hline & 2000 & $15.473^{\mathrm{bc}}$ & $202.5^{d}$ & $4.33^{\text {de }}$ & $185.833^{f}$ & $55.763^{f g}$ & $3.532^{\mathrm{abc}}$ \\
\hline & 2400 & $15.028^{\mathrm{bc}}$ & $200^{d}$ & $4.232^{\mathrm{efg}}$ & $200.75^{\mathrm{cd}}$ & $52.403^{g}$ & $3.107^{\mathrm{abc}}$ \\
\hline \multirow[t]{8}{*}{$\begin{array}{c}\text { Late } \\
\text { planting }\end{array}$} & Control & $12.335^{\mathrm{bc}}$ & $175^{\mathrm{d}}$ & $3.742^{\text {gh }}$ & $234.75^{\mathrm{a}}$ & $63.675^{\text {cde }}$ & $3.512^{\mathrm{abc}}$ \\
\hline & 400 & $14.403^{\mathrm{abc}}$ & $181.667^{\mathrm{d}}$ & $4.239^{\mathrm{efg}}$ & $218.5^{b}$ & $64.983^{\text {cde }}$ & $3.462^{\mathrm{abc}}$ \\
\hline & 800 & $15.048^{\mathrm{abc}}$ & $235^{c}$ & $4.259^{\mathrm{ef}}$ & $206.75^{c}$ & $70.745^{\mathrm{bc}}$ & $3.141^{\mathrm{abc}}$ \\
\hline & 1200 & $17.092^{\mathrm{ab}}$ & $252.5^{c}$ & $4.852^{\mathrm{bc}}$ & $204^{\text {cd }}$ & $73.35^{b}$ & $3.572^{\mathrm{abc}}$ \\
\hline & 1600 & $14.955^{\mathrm{bc}}$ & $237.5^{c}$ & $4.782^{\mathrm{bcd}}$ & $192.25^{\mathrm{ef}}$ & $65.983^{\text {bcd }}$ & $2.888^{c}$ \\
\hline & 2000 & $13.523^{c}$ & $202.5 d$ & $4.573^{\text {bcde }}$ & $198.25^{\text {de }}$ & $61.46^{\text {def }}$ & $3.789^{\mathrm{ab}}$ \\
\hline & 2400 & $12.35^{\text {bc }}$ & $140^{\mathrm{e}}$ & $3.743^{\text {fgh }}$ & $204.75^{\mathrm{cd}}$ & $54.62^{\mathrm{fg}}$ & $3.305^{\mathrm{abc}}$ \\
\hline & & ANOVA & & & & & \\
\hline date & & $* *$ & $* * *$ & $* * *$ & $* * *$ & n.s. & n.s. \\
\hline Priming & & n.s. & $* * *$ & $* * *$ & $* * *$ & $* * *$ & $*$ \\
\hline $\begin{array}{l}\text { date } \times \\
\text { priming }\end{array}$ & & $* *$ & $* * *$ & $* * *$ & $* * *$ & $* * *$ & * \\
\hline
\end{tabular}

* Within each column, different letters indicate significant differences at $\mathrm{P} \leq 0.05$ (Duncan test). n.s., $*, * *$ and $* * *$ indicate non-significant or significant differences at $\mathrm{P}$, $0.05,0.01$ or 0.001 , respectively. 
Table 3. Effect of priming by salicylic acid (SA) on, net photosynthetic rate $(\mathrm{PN})$, stomatal conductance (gs), Transpiration Rate (E), intercellular $\mathrm{CO}_{2}$ concentration (Ci), Carboxylation efficiency (CE), and photosynthetic water use efficiency (WUE) in heading stage in wheat plants.

\begin{tabular}{|c|c|c|c|c|c|c|c|}
\hline Planting date & $\begin{array}{c}\text { Priming } \\
{[\mu \mathrm{M}]}\end{array}$ & {$\left[\begin{array}{c}P_{N} \\
{\left[\mu \mathrm{mol}\left(\mathrm{CO}_{2}\right)\right.} \\
\left.\mathrm{m}^{-2} \mathrm{~s}^{-1}\right]\end{array}\right.$} & $\begin{array}{c}g_{s} \\
{\left[\mathrm{mmol} \mathrm{m}^{-}\right.} \\
\left.{ }^{2} \mathrm{~s}^{-1}\right]\end{array}$ & $\begin{array}{c}E \\
{\left[\mathrm{mmol}\left(\mathrm{H}_{2} \mathrm{O}\right)\right.} \\
\left.\mathrm{m}^{-2} \mathrm{~s}^{-1}\right]\end{array}$ & $\begin{array}{c}\mathrm{Ci} \\
{\left[\mu \mathrm{mol}\left(\mathrm{CO}_{2}\right)\right.} \\
\left.\operatorname{mol}(\mathrm{air})^{-1}\right]\end{array}$ & $\begin{array}{c}\mathrm{CE} \\
{\left[\mathrm{mmol}^{\left.-\mathrm{CO}_{2}\right)}\right.} \\
\left.\mathrm{m}^{-2} \mathrm{~s}^{-1}\right]\end{array}$ & $\begin{array}{c}\text { WUE } \\
{\left[\mu \mathrm{mol}\left(\mathrm{CO}_{2}\right)\right.} \\
\mathrm{mmol}^{-1} \\
\left.\left(\mathrm{H}_{2} \mathrm{O}\right)\right] \\
\end{array}$ \\
\hline \multicolumn{8}{|l|}{ Stress period } \\
\hline \multirow[t]{7}{*}{$\begin{array}{l}\text { Recommended } \\
\text { planting date }\end{array}$} & Control & $17.758^{\mathrm{gh}}$ & $345^{\mathrm{ef}}$ & $8.11^{\text {cde }}$ & $238.5^{\mathrm{ab}}$ & $78.79^{\text {hi }}$ & $1.945^{\mathrm{c}}$ \\
\hline & 400 & $21.535^{\mathrm{bc}}$ & $382.5^{\mathrm{de}}$ & $8.565^{\text {bcde }}$ & $225.733^{\mathrm{cd}}$ & $87.485^{\text {defg }}$ & $2.259^{\mathrm{abc}}$ \\
\hline & 800 & $22.187^{\mathrm{ab}}$ & $420^{\mathrm{cd}}$ & $9.77^{\mathrm{ab}}$ & $216.25^{\text {de }}$ & $99.410^{c}$ & $2.517^{\mathrm{a}}$ \\
\hline & 1200 & $23.553^{\mathrm{a}}$ & $537.5^{\mathrm{b}}$ & $10.225^{\mathrm{a}}$ & $209.5^{\mathrm{e}}$ & $111.79^{\mathrm{a}}$ & $2.001^{\mathrm{c}}$ \\
\hline & 1600 & $20.395^{\mathrm{bcd}}$ & $502.5^{\mathrm{b}}$ & $9.68^{\mathrm{ab}}$ & $210.5^{\mathrm{e}}$ & $108.79^{\mathrm{ab}}$ & $2.271^{\mathrm{abc}}$ \\
\hline & 2000 & $19.897^{\text {cde }}$ & $420^{\mathrm{cd}}$ & $8.735^{\text {bcde }}$ & $225.25^{\mathrm{cd}}$ & $103.09^{\mathrm{bc}}$ & $2.111^{\mathrm{bc}}$ \\
\hline & 2400 & $19.077^{\text {defg }}$ & $332.5^{\mathrm{ef}}$ & $7.910^{\mathrm{de}}$ & $234.233^{\mathrm{abc}}$ & $84.095^{\text {efgh }}$ & $2.305^{\mathrm{abc}}$ \\
\hline \multirow[t]{8}{*}{ Late planting } & Control & $17.052^{\mathrm{h}}$ & $307.5^{f}$ & $7.555^{\mathrm{e}}$ & $242.733^{\mathrm{a}}$ & $75.120^{\mathrm{i}}$ & $2.163^{\mathrm{abc}}$ \\
\hline & 400 & $17.967^{\mathrm{fgh}}$ & $332.5^{\mathrm{ef}}$ & $8.978^{\mathrm{abcd}}$ & $218^{\mathrm{de}}$ & $78.226^{\mathrm{hi}}$ & $2.165^{\mathrm{abc}}$ \\
\hline & 800 & $19.025^{\text {defg }}$ & $465^{\mathrm{bc}}$ & $9.058^{\mathrm{abcd}}$ & $216.25^{\text {de }}$ & $89.358^{\mathrm{def}}$ & $2.140^{\mathrm{bc}}$ \\
\hline & 1200 & $20.672^{\text {bcd }}$ & $622.5^{\mathrm{a}}$ & $9.58^{\mathrm{ab}}$ & $210.25^{\mathrm{e}}$ & $94.82^{\mathrm{cd}}$ & $2.278^{\mathrm{abc}}$ \\
\hline & 1600 & $19.69^{\text {def }}$ & $397.5^{\text {cde }}$ & $9.207^{\mathrm{abc}}$ & $217.25^{\mathrm{de}}$ & $90.58^{\mathrm{de}}$ & $2.135^{b c}$ \\
\hline & 2000 & $18.150^{\text {efgh }}$ & $355^{\text {def }}$ & $8.497^{\text {bcde }}$ & $218^{\mathrm{de}}$ & $81.025^{\text {fghi }}$ & $2.419^{\mathrm{ab}}$ \\
\hline & 2400 & $17.453^{\mathrm{gh}}$ & $327.5^{\mathrm{ef}}$ & $7.82^{\mathrm{de}}$ & $230.667^{\mathrm{bc}}$ & $80.36^{\text {ghi }}$ & $2.232^{\mathrm{abc}}$ \\
\hline & & ANOVA & & & & & \\
\hline Date & & $* * *$ & n.s. & n.s. & n.s. & $* * *$ & n.s. \\
\hline Priming & & $* * *$ & $* * *$ & $*$ & $* * *$ & $* * *$ & $*$ \\
\hline date $\times$ priming & & $* * *$ & $* * *$ & * & $* * *$ & $* * *$ & $*$ \\
\hline
\end{tabular}

* Within each column, different letters indicate significant differences at $\mathrm{P} \leq 0.05$ (Duncan test). n.s., *, ** and *** indicate non-significant or significant differences at $\mathrm{P}$, $0.05,0.01$ or 0.001 , respectively. 
Table 4. Effect of priming by salicylic acid (SA) on, net photosynthetic rate (PN), stomatal conductance (gs), Transpiration Rate (E), intercellular CO2 concentration (Ci), Carboxylation efficiency (CE), and photosynthetic water use efficiency (WUE) in grain filling stage in wheat plants.

\begin{tabular}{|c|c|c|c|c|c|c|c|}
\hline $\begin{array}{c}\text { Planting } \\
\text { date }\end{array}$ & $\begin{array}{c}\text { Priming } \\
{[\mu \mathrm{M}]}\end{array}$ & $\begin{array}{c}P_{N} \\
{\left[\mu \mathrm{mol}\left(\mathrm{CO}_{2}\right)\right.} \\
\left.\mathrm{m}^{-2} \mathrm{~s}^{-1}\right]\end{array}$ & $\begin{array}{c}g_{s} \\
{[\mathrm{mmol}} \\
\left.\mathrm{m}^{-2} \mathrm{~s}^{-1}\right]\end{array}$ & $\begin{array}{c}E \\
{\left[\mathrm{mmol}\left(\mathrm{H}_{2} \mathrm{O}\right)\right.} \\
\left.\mathrm{m}^{-2} \mathrm{~s}^{-1}\right] \\
\end{array}$ & $\begin{array}{c}\mathrm{Ci} \\
{\left[\mu \mathrm{mol}\left(\mathrm{CO}_{2}\right)\right.} \\
\left.\operatorname{mol}(\text { air })^{-1}\right] \\
\end{array}$ & \begin{tabular}{|c|}
$\mathrm{CE}$ \\
{$\left[\mathrm{mmol}^{-\left(\mathrm{CO}_{2}\right)}\right.$} \\
$\left.\mathrm{m}^{-2} \mathrm{~s}^{-1}\right]$ \\
\end{tabular} & $\begin{array}{c}\text { WUE } \\
{\left[\mu \mathrm{mol}\left(\mathrm{CO}_{2}\right)\right.} \\
\left.\mathrm{mmol}^{-1}\left(\mathrm{H}_{2} \mathrm{O}\right)\right]\end{array}$ \\
\hline $\begin{array}{l}\text { Stress } \\
\text { period }\end{array}$ & & & & & & & \\
\hline \multirow[t]{7}{*}{\begin{tabular}{|c|} 
Recomme \\
nded \\
planting \\
date \\
\end{tabular}} & Control & $7.53^{\mathrm{g}}$ & $77.5^{\text {de }}$ & $3.547^{\mathrm{bc}}$ & $226.583^{\mathrm{a}}$ & $39.593^{g}$ & $2.122^{b c}$ \\
\hline & 400 & $7.803^{\mathrm{fg}}$ & $80^{\text {de }}$ & $4.368^{b}$ & $214.417^{\mathrm{ab}}$ & $40.063^{f g}$ & $1.79^{\mathrm{a}}$ \\
\hline & 800 & $9.42^{\mathrm{ef}}$ & $82.5^{\text {de }}$ & $4.723^{b}$ & $206.75^{\mathrm{bc}}$ & $67^{\mathrm{bc}}$ & $1.788^{\mathrm{c}}$ \\
\hline & 1200 & $11.232^{\mathrm{cd}}$ & $165^{\mathrm{b}}$ & $4.918^{\mathrm{ab}}$ & $187.75^{\text {cde }}$ & $68.253^{\mathrm{bc}}$ & $3.416^{\mathrm{bc}}$ \\
\hline & 1600 & $10.655^{\text {de }}$ & $155^{b}$ & $4.712^{\mathrm{b}}$ & $172^{\text {ef }}$ & $56.16^{\text {de }}$ & $2.383^{b c}$ \\
\hline & 2000 & $9.082^{\text {efg }}$ & $102.5^{\mathrm{cd}}$ & $4.532^{\mathrm{b}}$ & $178.333^{\mathrm{ef}}$ & $48.77^{e}$ & $3.142^{\mathrm{bc}}$ \\
\hline & 2400 & $9.017^{\text {efg }}$ & $102.5^{\mathrm{cd}}$ & $4.252^{\mathrm{b}}$ & $197.75^{\text {bcd }}$ & $48.123^{\mathrm{ef}}$ & $2.283^{\mathrm{bc}}$ \\
\hline \multirow[t]{8}{*}{$\begin{array}{c}\text { Late } \\
\text { planting }\end{array}$} & Control & $11.947^{\mathrm{bcd}}$ & $140^{\mathrm{b}}$ & $2.292^{c}$ & $214^{\mathrm{ab}}$ & $61.9^{\mathrm{cd}}$ & $5.21^{\mathrm{bc}}$ \\
\hline & 400 & $13.053^{b}$ & $143.5^{b}$ & $3.842^{\mathrm{b}}$ & $198.75^{\text {bcd }}$ & $62.08^{\mathrm{cd}}$ & $2.267^{\mathrm{bc}}$ \\
\hline & 800 & $13.334^{\mathrm{b}}$ & $160^{\mathrm{b}}$ & $4.342^{\mathrm{b}}$ & $185.25^{\text {de }}$ & $76.623^{\mathrm{a}}$ & $2.716^{\mathrm{bc}}$ \\
\hline & 1200 & $15.607^{\mathrm{a}}$ & $205^{\mathrm{a}}$ & $6.267^{\mathrm{a}}$ & $165^{\mathrm{f}}$ & $78.6^{\mathrm{a}}$ & $1.809^{c}$ \\
\hline & 1600 & $12.728^{\mathrm{bc}}$ & $147.5^{\mathrm{b}}$ & $4.855^{\mathrm{b}}$ & $170.25^{\mathrm{ef}}$ & $71.133^{\mathrm{ab}}$ & $3.468^{\mathrm{b}}$ \\
\hline & 2000 & $12.305^{\mathrm{bcd}}$ & $110^{\mathrm{c}}$ & $3.745^{b}$ & $184.167^{\text {de }}$ & $63.663^{\mathrm{bcd}}$ & $2.215^{\mathrm{bc}}$ \\
\hline & 2400 & $8.852^{f g}$ & $67.5^{\mathrm{e}}$ & $3.572^{\mathrm{bc}}$ & $208^{\mathrm{ab}}$ & $32.14^{\mathrm{g}}$ & $2.479^{\mathrm{bc}}$ \\
\hline & & ANOVA & & & & & \\
\hline date & & $* * *$ & $* * *$ & n.s. & $*$ & $* * *$ & $*$ \\
\hline Priming & & $* * *$ & $* * *$ & ** & $*$ & $* * *$ & $* *$ \\
\hline $\begin{array}{c}\text { date } \times \\
\text { priming }\end{array}$ & & $* * *$ & $* * *$ & * & $* * *$ & $* * *$ & $* *$ \\
\hline
\end{tabular}

* Within each column, different letters indicate significant differences at $\mathrm{P} \leq 0.05$ (Duncan test). n.s., *, ** and *** indicate non-significant or significant differences at $\mathrm{P}$, $0.05,0.01$ or 0.001 , respectively. 
This increment may be due to more reduction in $E$ than to $P_{\mathrm{N}}$ in this phenological stage because of cool temperature in tillering stage. With some exceptions WUE was decreased by priming (Table 2, 3 and 4). Exceptions was found in late planting treatments in tillering and recommended planting in heading. It seems priming by SA enhanced water status of plants and RWC (Abdollahi and Shekari, 2013). It is reasonable from $E$ and $g_{s}$ data which increased in SA treatments compared to control treatments. Therefore, transpiration rate increased by SA application more than $P_{N}$ and WUE relatively decreased. Although, in our experiment WUE decreased by seed priming with SA, increasing in WUE by pretreatment with SA reported previously (Liu et al., 2011; Fariduddin et al., 2003; Khan et al., 2003).

\section{Chlorophyll Index and Chlorophyll Content}

In all growth stages first planting date had higher chlorophyll content index (CCI) compared to second planting date. Although, in first planting date heading stage had highest value among other phenological stages, but in late planting the highest amount of CCI was found in grain filling (Table 5). The lowest value among three growth stages was observed in tillering stage and second planted plants. In general, seed priming with $1200 \mu \mathrm{M}$ of SA had greatest impact on increasing the amount of CCI in both planting date and in all three growth stages. The lowest values was observed control treatments and in late planting date.

Like CCI, chlorophyll a and b were affected by seed priming and planting date treatments (Table 5). The amounts of $\mathrm{Chl} \mathrm{a,} \mathrm{b,} \mathrm{total} \mathrm{and} \mathrm{a/b} \mathrm{were} \mathrm{increased}$ through $1200 \mu \mathrm{M}$ of SA treatment relative to control treatments in both planting dates. The amount of Chl b was increased to $10.5 \%$ through $2000 \mu \mathrm{M}$ of SA compared to control treatment in late planting. Overall, the amounts of $\mathrm{Chl}$ a and $\mathrm{b}$ on the first planting date were higher than second planting date. However, priming through SA could discount the adverse effects of late planting and the amount of chlorophyll reduction. It seems that the effect of SA on biosynthesis and/or protection of chlorophyll a are more than chlorophyll $b$. Because the ratio of chlorophyll $\mathrm{a} / \mathrm{b}$ in primed treatments in both planting dates are more than control treatments.

Chlorophyll content is important in maintenance of photosynthetic capacities (Jiang and Huang, 2001) and a key factor in determination of photosynthesis rate and dry matter production (dos Santos et al., 2013). Also, it is stated chlorophyll content is the most reliable parameter to estimate leaf growth and development (Albert et al. 2012). Gunes et al. (2007) reported in maize plants salt stress or application of SA had not significant effect on chlorophyll a, b and total content, but SA reduced carotenoids contentment. In contrast, Arfan et al. (2007) stated salinity decreased chlorophyll content of wheat, but SA increased chlorophyll content. Similarly, Sinha et al. (1993) pointed out that chlorophyll and carotenoid contents of maize were increased upon treatment with SA. Treatment with $500 \mu \mathrm{M}$ SA for $24 \mathrm{~h}$ before exposure to chilling provided protection on Rubisco activity and chlorophyll content (Yordanova and Popova, 
2007). It seems that this effect of SA on photosynthetic pigments depends to types of species, cultivar, method of SA application and its concentrations.

\section{Total dry weight (TDW)}

In comparison of two planting date, first planting had more dry weight compared to second planting date. With some exceptions, in all growth stages in treatments which had higher photosynthetic rates, higher accumulation of dry matter was found (Table 2, 3, 4 and 5).

Table 5. Effect of priming by salicylic acid (SA) on, Chlorophyll content index (CCI) in three stages, chlorophyll (Chl) (a), (b), (a/b), (a+b) content, and Biological Yield (BY) at harvest.

\begin{tabular}{|c|c|c|c|c|c|c|c|c|c|}
\hline Planting date & $\mid \begin{array}{c}\text { Priming } \\
{[\mu \mathrm{M}]}\end{array}$ & $\begin{array}{c}\text { CCI } \\
\text { (Tillering) }\end{array}$ & $\begin{array}{c}\text { CCI } \\
\text { (Heading) }\end{array}$ & $\begin{array}{c}\text { CCI } \\
\text { (Grain } \\
\text { filling) }\end{array}$ & $\begin{array}{c}\text { Chl } a \\
{\left[\mathrm{mg} \mathrm{g}^{-1}\right]}\end{array}$ & $\begin{array}{c}\text { Chl } b \\
{\left[\mathrm{mg} \mathrm{g}^{-1}\right]}\end{array}$ & $\begin{array}{l}\text { Chl } a+b \\
{\left[\mathrm{mg} \mathrm{g}^{-1}\right]}\end{array}$ & Chl $a / b$ & $\begin{array}{c}\mathrm{BY} \\
{\left[\mathrm{g} \mathrm{m}^{-2}\right]}\end{array}$ \\
\hline \multicolumn{10}{|l|}{ Stress period } \\
\hline \multirow[t]{7}{*}{$\begin{array}{c}\text { Recommended } \\
\text { planting date }\end{array}$} & Control & $16.740^{\mathrm{bc}}$ & $41.33^{\text {cd }}$ & $39.703^{\text {def }}$ & $0.8315^{\mathrm{e}}$ & $0.3762^{\mathrm{abc}}$ & $1.208^{\mathrm{ef}}$ & $2.273^{\text {de }}$ & $1237^{\text {de }}$ \\
\hline & 400 & $17.748^{\mathrm{bc}}$ & $44.76^{\mathrm{ab}}$ & $41.715^{\text {cd }}$ & $0.8543^{\mathrm{e}}$ & $0.2936^{\text {ef }}$ & $1.148^{\mathrm{fg}}$ & $2.910^{\mathrm{ab}}$ & $1437^{\mathrm{bc}}$ \\
\hline & 800 & $22.4^{\mathrm{ab}}$ & $45.533^{\mathrm{ab}}$ & $46.38^{\mathrm{b}}$ & $0.9573^{\mathrm{cd}}$ & $0.3913^{\mathrm{a}}$ & $1.349^{\mathrm{c}}$ & $2.447^{\mathrm{cd}}$ & $1572^{\mathrm{ab}}$ \\
\hline & 1200 & $28.210^{\mathrm{a}}$ & $46.85^{\mathrm{a}}$ & $48.95^{\mathrm{a}}$ & $1.16^{\mathrm{a}}$ & $0.4032^{\mathrm{a}}$ & $1.564^{\mathrm{a}}$ & $2.875^{\mathrm{ab}}$ & $1641^{\mathrm{a}}$ \\
\hline & 1600 & $26.922^{\mathrm{a}}$ & $46.313^{\mathrm{a}}$ & $41.163^{\mathrm{cd}}$ & $1.084^{\mathrm{b}}$ & $0.3843^{\mathrm{ab}}$ & $1.469^{\mathrm{b}}$ & $2.843^{\mathrm{abc}}$ & $1605^{\mathrm{a}}$ \\
\hline & 2000 & $23.52^{\mathrm{ab}}$ & $43.32^{\mathrm{bc}}$ & $38.893^{\mathrm{ef}}$ & $0.9434^{\mathrm{d}}$ & $0.3030^{\mathrm{ef}}$ & $1.246^{\mathrm{de}}$ & $3.114^{\mathrm{a}}$ & $1549^{\mathrm{ab}}$ \\
\hline & 2400 & $23.160^{\mathrm{ab}}$ & $39.537^{\text {de }}$ & $36.07^{\text {gh }}$ & $0.986^{\text {cd }}$ & $0.331^{\text {cdef }}$ & $1.317^{\mathrm{cd}}$ & $2.979^{\mathrm{ab}}$ & $1322^{\mathrm{cd}}$ \\
\hline \multirow[t]{8}{*}{ Late planting } & Control & $5.342^{\mathrm{e}}$ & $28.53^{\mathrm{ef}}$ & $33.923^{\mathrm{h}}$ & $0.751^{\mathrm{fg}}$ & $0.3372^{\text {bcde }}$ & $1.088^{\mathrm{gh}}$ & $2.228^{\text {de }}$ & $969^{f}$ \\
\hline & 400 & $5.96^{\text {de }}$ & $36.173^{\text {ef }}$ & $34.2^{\mathrm{h}}$ & $0.7965^{\mathrm{ef}}$ & $0.3106^{\text {ef }}$ & $1.107^{\mathrm{gh}}$ & $2.575^{\mathrm{bcd}}$ & $1237^{\text {de }}$ \\
\hline & 800 & $10.183^{\text {cde }}$ & $37.903^{h}$ & $39.83^{\mathrm{de}}$ & $0.7135^{\mathrm{g}}$ & $0.3211^{\text {def }}$ & $1.035^{\mathrm{h}}$ & $2.226^{\mathrm{de}}$ & $1281^{d}$ \\
\hline & 1200 & $14.01^{\mathrm{cd}}$ & $39.36^{\text {de }}$ & $43.105^{c}$ & $1.031^{\mathrm{bc}}$ & $0.3234^{\text {cdef }}$ & $1.355^{\mathrm{c}}$ & $3.203^{\mathrm{a}}$ & $1284^{\mathrm{d}}$ \\
\hline & 1600 & $4.805^{\mathrm{e}}$ & $37.35^{\mathrm{ef}}$ & $41.533^{\mathrm{cd}}$ & $0.8415^{\mathrm{e}}$ & $0.2841^{\mathrm{f}}$ & $1.126^{\mathrm{fgh}}$ & $2.963^{\mathrm{ab}}$ & $1188^{\text {de }}$ \\
\hline & 2000 & $4.433^{\mathrm{e}}$ & $32.333^{\mathrm{g}}$ & $38.403^{\mathrm{ef}}$ & $0.747^{\mathrm{fg}}$ & $0.3726^{\text {abcd }}$ & $1.120^{\mathrm{fgh}}$ & $2.008^{\mathrm{e}}$ & $1105^{\text {ef }}$ \\
\hline & 2400 & $4.09^{e}$ & $31.46^{\mathrm{g}}$ & $37.603^{\mathrm{fg}}$ & $0.628^{\mathrm{h}}$ & $0.2911^{\text {ef }}$ & $0.919^{\mathrm{i}}$ & $2.174^{\text {de }}$ & $1096^{\text {ef }}$ \\
\hline & & ANOVA & & & & & & & \\
\hline date & & $* * *$ & $* * *$ & $* * *$ & $* * *$ & $* * *$ & $* * *$ & $* * *$ & $* * *$ \\
\hline Priming & & n.s. & $* * *$ & $* * *$ & $* * *$ & $* *$ & $* * *$ & $* * *$ & $* * *$ \\
\hline date $\times$ priming & & $* *$ & $* * *$ & $* * *$ & $* * *$ & $* * *$ & $* * *$ & $* * *$ & n.s. \\
\hline
\end{tabular}

* Within each column, different letters indicate significant differences at $\mathrm{P} \leq 0.05$ (Duncan test). n.s., *, ** and $* * *$ indicate non-significant or significant differences at $\mathrm{P}$, $0.05,0.01$ or 0.001 , respectively 
Increment in fresh and dry weight of plants by SA treatment may due to increase in cellular dividing rate in apical meristem of root and shoot of plants which enhance plant growth (Sakhabutdinova et al., 2003). Horvath et al. (2007) reported in wheat seedlings SA enhanced growth rate via increasing auxins and cytokinins concentrations. Also, Agami (2013) stated higher rate of dry matter production of maize plants both in normal and salt stress conditions by application of SA due to induction of antioxidant enzymes activities, proline and photosynthetic pigments. Increasing dry weight of artichoke plants by application of SA reported by Rajabi et al (2013) Fariduddin et al. (2003). On the other hand, Singh and Usha (2003) showed high concentrations of SA have preventive effects on wheat and maize growth. In our experiment seed priming with 2000 and $2004 \mu \mathrm{M}$ SA concentrations had unfavorable effects on most recorded traits.

\section{CONCLUSIONS}

Presented results showed that change in planting date could affect photosynthetic parameters. In late planted plants PN was lower than conventional planting in tillering and heading stages, but in grain filling stage this trend was reversed. This trend approximately was found in gs and E traits. The highest values for PN, gs, E and CE were obtained in heading and then in tillering stages. In contrast, $\mathrm{Ci}$ was lower in heading stage and highest in grain filling stage. It seems that, in heading and flowering stage due to higher demand for photoassimilates PN showed higher values and in grain filling stage by aging of leaves capacity of photosynthesis relatively decreased. Seed priming by SA significantly increased PN and related parameters. Furthermore, the highest and lowest of WUE were achieved in tillering and heading, respectively. May be due to low temperature in early spring, the value of $E$ was lower than other stages and this affect WUE in this stage. Seed priming with SA decreased WUE compared to control treatment in both planting dates. As shown by gs, it seems SA induced to more opening the stoma and therefore increment in E was more than PN. According to results, seed pretreatment with $1200 \mu \mathrm{M}$ SA had appropriate performance than other SA concentrations.

\section{REFERENCES}

Abdolahi, M. and Shekari, F. 2013. Effect of priming by salicylic acid on vigor and performance of wheat seedlings at different planting dates. Cereal Research. 3: 1732. [In Persian with English Abstract].

Agami, R. 2013. Alleviating the adverse effects of $\mathrm{NaCl}$ stress in maize seedlings by pretreating seeds with salicylic acid and 24-epibrassinolide. South African Journal of Botany 88 171-177.

Alijani, B. 2006. Climatology of Iran. 5th ed. Payam Noor University Pub. Tehran. Iran. [In Persian].

Albert, B., Le Cahe'rec, F., Niogret, M., Faes, P., Avice, J., Leport, L. and Bouchereau, A. 2012. Nitrogen availability impacts oilseed rape (Brassica napus L.) plant water status and proline production efficiency under water-limited condition. Planta. 236: 659-676 
Arfan, M., Athar, H.R., Ashraf, M. 2007. Does exogenous application of salicylic acid through the rooting medium modulate growth and photosynthetic capacity in two differently adapted spring wheat cultivars under salt stress? Journal of Plant Physiology. 164: 685-694.

Ashraf. M., Karim. F., and Rasul. E, 2002. Interactive effects of gibberellic acid and salt stress on growth, ion accumulation and photosynthetic capacity of two-spring wheat (Triticum aestivum L.) cultivars differing in salt tolerance. Plant Growth Regulation. 36: 49-59.

Clark. J. M., and Mc ciag. T. N, 1982. Evaluation of techniques for screening for drought resistance in wheat. Crop Science. 22: 503-505.

dos Santos, E.F., Zanchim, B.J., de Campos, A.G., Garrone, R.F. and Junior, J.L. 2013. Photosynthesis rate, chlorophyll content and initial development of nut without micronutrient fertilization. Revista Brasileira de Cienia dosolo.37: 1334-1342.

Fariduddin. Q., Hayat. S., and Ahmad. A, 2003. Salicylic acid influences net photosynthetic rate, carboxylation efficiency, nitrate reductase activity, and seed yield in Brassica juncea. Photosynthetica. 41:281-284.

Fitter, A. and Hey, RKM. 2012. Environmental physiology of plants. (3rd Ed.). Acad Press. UK.

Gunes, A., Inal, A., Alpaslan, M, Eraslan, F, Guneri Bagci, E and Cicek, N. 2007. Salicylic acid induced changes on some physiological parameters symptomatic for oxidative stress and mineral nutrition in maize (Zea mays L.) grown under salinity. Journal of Plant Physiology 164: 728-736.

Hejnák, V., Hniličková, H. and Hnilička, F. 2014. Effect of ontogeny, heterophylly and leaf positionon the gas exchange of the hop plant. Plant, Soil and Environment. 60: 525-530.

Horvath, E., Pal, M., Szalai, G., Paldi, E. and Janda, T. 2007. Exogenous 4-hydrobenzoic acid and salicylic acid modulate the effect of short term drought and freezing stress on wheat plants. Biologia Plantarum. 51: 480-487.

Janda, T., Gondor, O.K., Yordanova, R., Sazalai, G. and Pal, M. 2014. Salicylic acid and photosynthesis: Signaling and effects. Acta Physiologia Plantarum. 36: 25372546.

Jiang, Y. and Huang. B. 2001. Responses of photosynthesis and water relations to heat stress alone or in combination with drought: A comparison of tall fescue and perennial ryegrass. HortScience 36: 682-686.

Khan, W., B. Prithviraj, and D. L. Smith. 2003. Photosynthetic responses of corn and soybean to foliar application of salicylates. Journal of Plant Physiology. 160: 485492.

Khodary, S. E. A, 2004. Effect of salicylic acid on growth, photosynthesis and carbohydrate metabolism in salt-stressed maize plants. International Journal of Agriculture and Biology. 6:5-8.

Liu, W., Ai, X. Z., Liang, W. J., Wang, H. T., Liu, S. X., \& Zheng, N. (2009). Effects of salicylic acid on the leaf photosynthesis and antioxidant enzyme activities of cucumber seedlings under low temperature and light intensity. Chinese Journal of Applied Ecology, 20, 441-445.

Liu. C., Guo. J., Cui. Y., Lu. T., Zhang. X., Shi. G, 2011. Effects of cadmium and salicylic acid on growth, spectral reflectance and photosynthesis of castor bean seedlings. Plant and Soil. 344: 131-141.

Meidner. H, 1984. Class experiments in plant physiology. Unwin Hyman Co London. Pp. 53-54.

Nazar, R, Iqbal, N., Syeed, S. and Khan, NA. 2011. Salicylic acid alleviates decreases in photosynthesis under salt stress by enhancing nitrogen and sulfur assimilation and 
antioxidant metabolism differentially in two mungbean cultivars. Journal of Plant Physiology 168: 807-815

Nebauer, S.G., Renau-Morata, B., Guardiola, J.L. and Molina, R.V. 2011. Photosynthesis down-regulation precedes carbohydrate accumulation under sink limitation in Citrus. Tree Physiology. 31: 169-177

Poor, P., Gémes, K., Horváth, F., Szepesi, A., Simon, M.L. and Tari, I. 2011. Salicylic acid treatment via the rooting medium interferes with stomatal response, CO2 fixation rate and carbohydrate metabolism in tomato, and decreases harmful effects of subsequent salt stress. Plant Biology. 13: 105-114.

Poor, P. and Tari, I. 2012. Regulation of stomatal movement and photosynthetic activity in guard cells of tomato abaxial epidermal peels by salicylic acid. Functional Plant Biology 39: 1028-1037.

Rajabi, M., Ahmadian, M. and Kalvandi, R. 2013. Effects of salinity and salicylic acid pretreatment on germination and seedling growth of Artichoke (Cynara scolymus L.). Caspian Journal of Applied Sciences Research. 2: 1-7.

Raskin. I, 1992. Role of salicylic acid in plants. Annual Review of Plant Physiology and Plant Molecular Biology. 43:439-463.

Ribeiro, R.V., Machado, E.C., Santos, M.G. and Oliveira, R.F. 2009. Photosynthesis and water relations of well-watered orange plants as affected by winter and summer conditions. Photosynthetica. 47: 215-222.

Sakhabutdinova, A.R., Fatkhudinova, D.R., Bezorukova, M.V. and Shakirova, F.M. 2003. Salicylic acid prevents damage action of stress factors on wheat plants. Bulgarian Journal of Plant Physiology. 1: 314-319.

Sandoval-Yepiz, M.R., 2004. Reguladores de crecimiento XXIII: Efecto del á́cido salici'lico en la biomasa del cempazu'chitl (Tagetes erecta). In: Tesis de Licenciatura, Instituto Tecnológico Agropecuario, Conkal, Yucatán, México.

Sinha, S. K., H. S. Srivastava and R. D. Tripathi, 1993. Influence of some growth regulators and cations on inhibitions of chlorophyll biosynthesis by lead in maize. Bulletin of Environmental Contamination and Toxicology. 51- 241-246.

Singh. B., and Usha. K, 2003. Salicylic acid induced physiological and biochemical changes in wheat seedling under water stress. Plant Growth Regulation. 39: 137141.

Wang, R.H., Chang, J.C., Li, K.T., Lin, T.S. and Chang, L.S. 2014. Leaf age and light intensity affect gas exchange parameters and photosynthesis within the developing canopy of field net-house-grown papaya trees. Scientia Horticulturae. 165: 365373.

Yordanova. R., Popova. L, 2007. Effect of exogenous treatment with salicylic acid on photosynthetic activity and antioxidant capacity of chilled wheat plants. General and Applied Plant Physiology. 33: 155-170.

Zhen. F., Fang. L., Cheng-shu. Z., Huai-riu. S., Xian-zhi. S., Yong-kweon. Y, 2010. Effects of acetylsalicylic acid and calcium chloride on photosynthetic apparatus and reactive oxygen-scavenging enzymes in Chrysanthemum under low temperature stress with low light. Agricultural Sciences in China. 9 (12): 17771786. 